\title{
Endoplasmic reticulum stress in lung disease
}

\author{
Stefan J. Marciniak
}

Affiliation: Cambridge Institute for Medical Research, University of Cambridge, Cambridge, UK.

Correspondence: Stefan J. Marciniak, University of Cambridge, Cambridge Institute for Medical Research, Hills Road, Cambridge, CB2 OXY, UK. E-mail: sjm20Acam.ac.uk

$@$ ERSpublications

Endoplasmic reticulum stress may play a role in pathogenesis of pulmonary fibrosis and cancer http://ow.ly/Ys5k30bRmL5

Cite this article as: Marciniak SJ. Endoplasmic reticulum stress in lung disease. Eur Respir Rev 2017; 26: 170018 [https://doi.org/10.1183/16000617.0018-2017].

ABSTRACT Exposure to inhaled pollutants, including fine particulates and cigarette smoke is a major cause of lung disease in Europe. While it is established that inhaled pollutants have devastating effects on the genome, it is now recognised that additional effects on protein folding also drive the development of lung disease. Protein misfolding in the endoplasmic reticulum affects the pathogenesis of many diseases, ranging from pulmonary fibrosis to cancer. It is therefore important to understand how cells respond to endoplasmic reticulum stress and how this affects pulmonary tissues in disease. These insights may offer opportunities to manipulate such endoplasmic reticulum stress pathways and thereby cure lung disease.

\section{Introduction}

The crowded molecular environment of a cell's interior poses a problem to protein synthesis. As a globular protein is made, its polypeptide chain must be folded into a specific final conformation. This involves stages when amino acids normally buried within the protein's core are exposed. These residues are available to interact with other proteins and could lead to aggregation. For this reason, cells possess molecular chaperones whose function is to bind incompletely folded proteins and shield them from inappropriate interactions with neighbouring molecules [1]. Although peptide bonds are made by ribosomes in the cytoplasm, proteins destined for secretion or insertion into membranes are folded inside a membrane-bound compartment called the endoplasmic reticulum (ER). This organelle permits proteins to undergo modifications, including glycosylation and disulphide bond formation, that could not occur in the cytosol. The ER therefore contains high concentrations of dedicated chaperones and enzymes that catalyse the folding of its client proteins. However, such an arrangement creates its own challenges, not least of which is to ensure the activity of ribosomes in the cytoplasm matches the capacity of the ER to fold the resulting load of new proteins.

If the rate at which new proteins enter the ER exceeds its folding capacity, there is a threat that aggregation will occur. It is this threat to overwhelm the protein folding machinery that is heuristically termed "ER stress". The cellular processes that defend against ER stress are collectively called the unfolded protein response (UPR) [2] (figure 1). This multifaceted process combines mechanisms to reduce the activity of ribosomes with mechanisms to increase the protein-folding capacity of the ER. In parallel,

Received: March 082017 | Accepted after revision: May 152017

Support statement: Stefan J. Marciniak has received funding from Medical Research Council UK (G1002610), Alpha 1 Foundation, British Lung Foundation and Papworth Hospital NHS Foundation Trust. Funding information for this article has been deposited with the Crossref Funder Registry.

Conflict of interest: None declared.

Provenance: Commissioned article, peer reviewed.

Copyright CERS 2017. ERR articles are open access and distributed under the terms of the Creative Commons Attribution Non-Commercial Licence 4.0. 


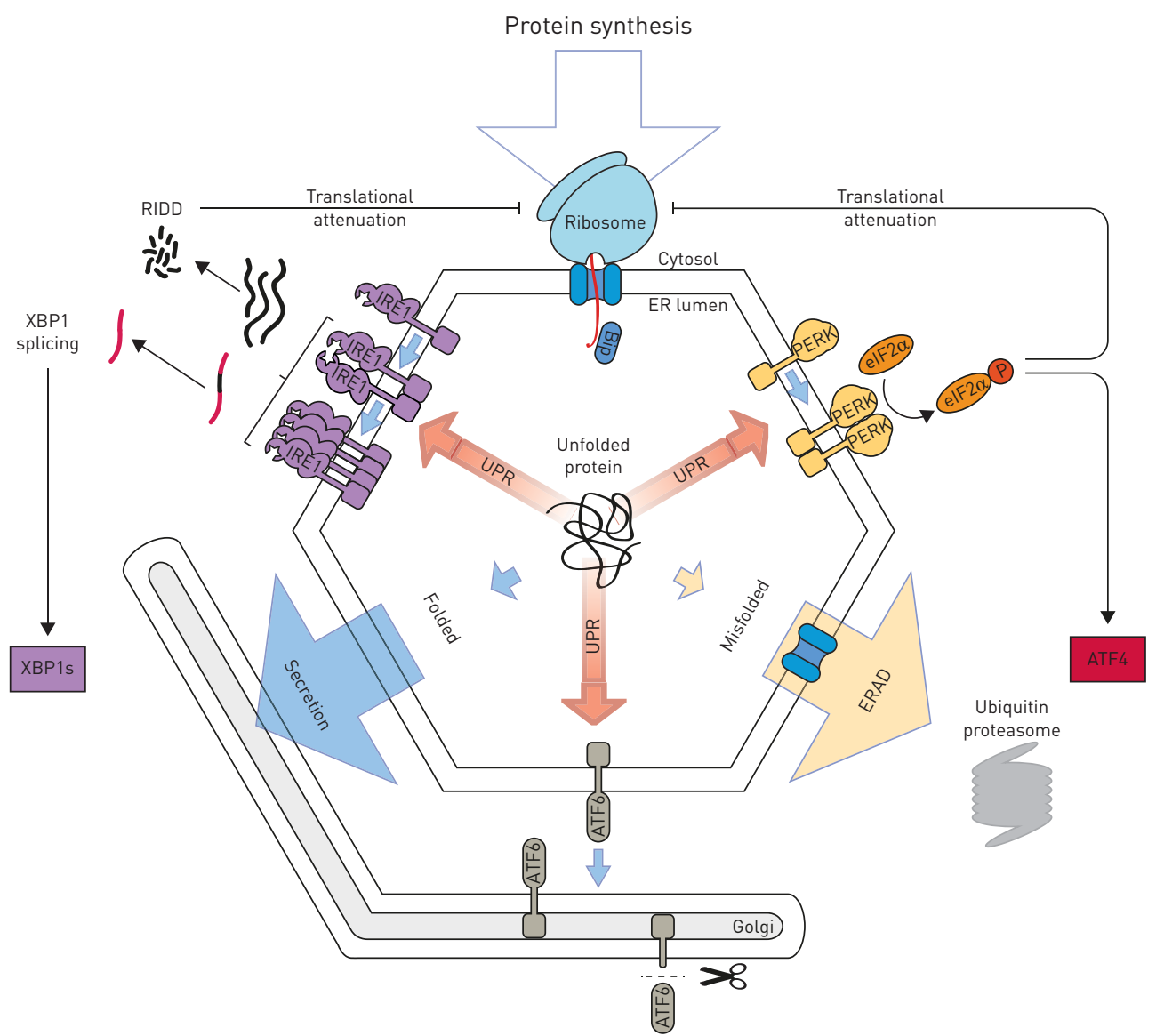

FIGURE 1 Proteostasis in the endoplasmic reticulum (ER). Proteins enter the ER and rapidly associate with chaperones such as binding immunoglobulin protein (BiP). Once correctly folded, proteins continue along the secretory pathway via the Golgi apparatus. Proteins that fail to fold correctly are retained in the ER and eventually targeted for proteasomal degradation by ER-associated degradation (ERAD). The threatened accumulation of unfolded proteins in the ER activates the unfolded protein response (UPR) via inositolrequiring enzyme (IRE)1, ATF6 and PERK. IRE1 initiates splicing of mRNA encoding the transcription factor XBP1; ATF6 activation leads to its proteolytic processing in the Golgi apparatus and the release of a soluble transcription factor domain. Both XBP1 and cleaved ATF6 upregulate components of the ER folding and degradation machineries. Activated PERK phosphorylates elF2 $\alpha$ to inhibit most translation, but increases the translation of ATF4. Targets of ATF4 promote oxidative folding, amino acid synthesis and recovery of normal translation rates. In addition, IRE1 reduces translation during stress, by degrading ER-localised mRNAs in a process termed regulated IRE1-dependent decay (RIDD). Reproduced and modified from [1] with permission.

terminally misfolded proteins are identified and returned to the cytoplasm for degradation by the ubiquitin proteasome system. This ER-associated degradation is augmented by the UPR and is responsible for the continued removal of mutated secretory proteins from the cell. If these protective processes fail and ER stress cannot be resolved, death pathways are activated. In addition, ER stress can trigger inflammatory signalling and modulate differentiation status, both of which contribute to the development of lung disease. It is often not appreciated that the ER stress itself is very rarely measured directly. Since ER stress refers to the incipient failure of protein folding, which is a highly complex condition, we instead tend to measure the cellular responses to it. That is, the UPR is used as a surrogate for ER stress, but it is important to bear in mind that this presupposes that the sensors of the UPR are functioning optimally.

In this mini-review, we examine each ER stress sensor in turn and then discuss their relevance to pulmonary fibrosis and cancer, the two lung conditions in which the UPR has been studied most thoroughly. A more comprehensive list of pulmonary disorders in which ER stress has been implicated is provided in table 1.

\section{UPR sensors}

ER stress is not solely a pathological phenomenon. Even healthy cells experience it when switching from low to high rates of secretion [1]. For this reason, the sensors of the UPR are part of everyday protein 
TABLE 1 Pulmonary disorders linked with endoplasmic reticulum stress

homeostasis (often called "proteostasis") and are necessary to fine-tune the secretory pathway to ensure a sufficient, but not wastefully excessive, protein-folding capacity. Three classes of transmembrane protein monitor the ER luminal environment and transmit their signals to the rest of the cell using three different second messengers.

Inositol-requiring enzyme (IRE) 1 is the oldest ER stress sensor in evolutionary terms (figure 1). Mammals possess two isoforms. IRE1 $\alpha$ is the ubiquitous form found in all cells that possess an ER, while IRE1 $\beta$ is restricted to the cells of the gut and lung and appears to play a specific function in mucus production. When sufficient protein-folding capacity exists in the ER, IRE1 is in an inactive state bound by the ER chaperone binding immunoglobulin protein (BiP). During ER stress, BiP dissociates to permit IRE1 to self-associate into large oligomers, but some evidence hints at a possible direct interaction between IRE1 and misfolding proteins in the ER lumen. IRE1 contains a kinase domain, but rather than phosphorylate other substrates, IRE1 autophosphorylates as part of its activation process. This triggers its RNA endonuclease activity, which is critical for signalling to the nucleus.

Activated IRE1 selectively cleaves the mRNA encoding a transcription factor called XBP1. The primary transcript of XBP1 encodes an inactive protein. During ER stress, IRE1 cuts either side of a 22-nucleotide intron and following re-ligation by RtcB the mRNA of the newly spliced XBP1 generates a potent transcription factor that induces genes of the UPR. This unique splicing event depends on the frameshift caused by excision of the intron, but it has been noted that IRE1 has additional beneficial effects during ER stress, even in cells lacking XBP1. Part of this XBP1-independent effect involves a second, less selective endonuclease activity that degrades many mRNAs associated with the ER membrane. This regulated IRE1-dependent decay (RIDD) rids the cell of mRNAs encoding secretory proteins, which helps to relieve the demand for ER chaperones by reducing the synthesis of secretory proteins. IRE1 has an additional function independent of its endonuclease activity. In a manner analogous to some cell surface receptor kinases, activated IRE1 acts as a platform for the assembly of signalling complexes via the scaffold protein TRAF2 leading to activation of the JNK signalling pathway, which can variously initiate inflammatory or apoptotic end-points depending upon context.

ATF6a is the prototypical member of a second class of ER stress sensor. Many paralogues exist, each with different tissue expression patterns and probably differing target gene profiles. ATF6a is another ER transmembrane protein that is held inactive by binding to BiP. Dissociation from BiP during ER stress is the primary mechanism for its activation, although it is also sensitive to alterations of the ER redox environment, which may contribute to its activation. Once released from its inactive BiP-bound state, ATF6 traffics to the Golgi apparatus where it is cleaved by two proteases, S1P and S2P, which liberate a soluble transcription factor that helps to induce UPR target genes (figure 1).

PERK activates the third arm of the UPR. It is regulated in a manner similar to IRE1, but once activated PERK recruits and phosphorylates the translation initiation factor eIF2 $\alpha$ [38] (figure 1). Reports that PERK can also phosphorylate the transcription factor Nrf2 must be treated with some caution because induction of PERK's downstream target genes is entirely dependent on phosphorylation of eIF2 $\alpha$ [39]. This phosphorylation serves two functions: it inhibits protein synthesis directly, while simultaneously triggering a gene expression programme via the transcription factor ATF4 [38, 40]. Because several kinases converge to phosphorylate eIF $2 \alpha$ in response to diverse stresses, the downstream pathway has been named the integrated stress response (ISR). It is therefore important not to assume that phosphorylation of eIF2 $\alpha$ or activation of its downstream response must always imply ER stress; the specific kinase involved should be determined.

Targets of ATF4 include antioxidant genes and genes involved in amino acid sufficiency [41]. ATF4 induces ATF3 and CHOP, transcription factors that contribute to the induction of GADD34 (also known as PPP1R15A), a master regulator of the ISR that dephosphorylates eIF2 $\alpha$ to restore normal levels of 


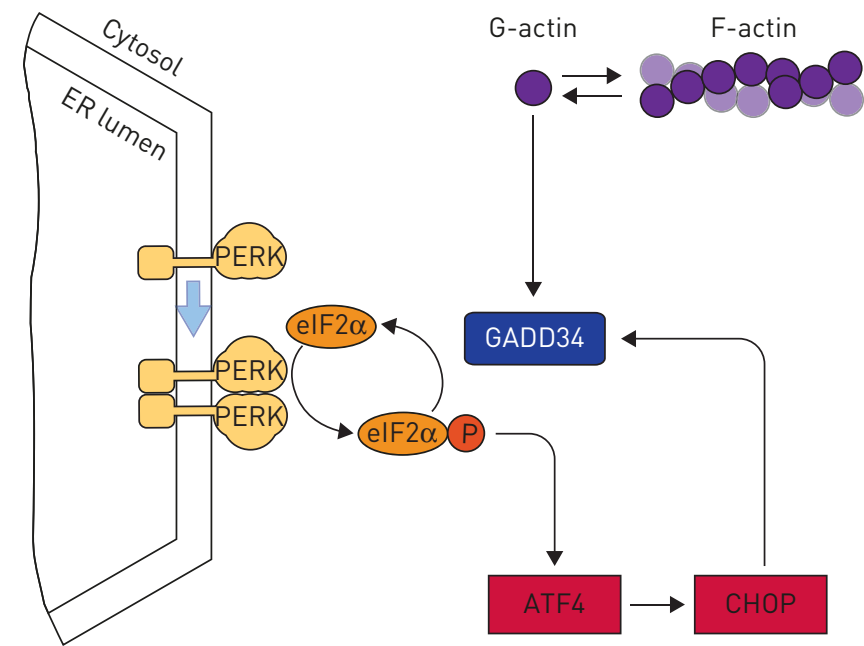

FIGURE 2 GADD34 is a master regulator of the integrated stress response. Phosphorylation of elF2 $\alpha$ increases the translation of ATF4. ATF4 transcriptionally induces CHOP, which induces expression of GADD34 (also known as PPP1R15A). In the presence of sufficient levels of G-actin, GADD34 will form a complex with PP1 and actin which selectively dephosphorylates elF2 $\alpha$. Factors that modulate actin polymerisation will therefore tune the sensitivity of the cell to activation of elF2 $\alpha$ kinases including the endoplasmic reticulum (ER) stress sensor PERK.

protein synthesis [40] (figure 2). GADD34 appears to be regulated both at the transcriptional level and by factors that regulate the actin cytoskeleton [42]. Since the assembly of actin fibres is sensitive to growth signals, GADD34 offers a potential mechanism to further integrate ISR and mitogenic signals.

Dephosphorylation of eIF2 $\alpha$ is necessary for the resumption of protein synthesis following its inhibition during the immediate response to stress. This later recovery of protein synthesis permits the expression of the antioxidant genes that by then have been induced by ATF4. This later response is necessary for cells to tolerate ER stress-induced reactive oxygen species. CHOP is frequently described simplistically as a "pro-apoptotic" factor, but again caution is warranted. The majority of CHOP's targets have no clear link with apoptosis, but rather appear to defend ongoing protein secretion, and in some disease models loss of CHOP causes increased cell death [43]. This apparent paradox, with the recovery of translation contributing to both survival and death, remains to be fully elucidated. One plausible explanation is that recovery of protein synthesis is pro-survival during responses to transient ER stress, but becomes toxic during chronic ER stress, when ongoing protein synthesis overwhelms the adaptive potential of the system. Evidence has implicated these pathways in the responses to oxidative stress imposed by cigarette smoke, but this requires further exploration [25-28]. Although ATF4 signalling is activated in the airways of many patients with chronic obstructive pulmonary disease [44], a direct mechanistic link with disease pathogenesis remains elusive.

\section{Cancer}

UPR signalling via PERK is necessary for the growth of large solid tumours in mice, while pharmacological inhibition of PERK has potent anticancer effects [45-47]. PERK has been implicated in tumour resistance to radiotherapy [48] and PERK-ATF4-mediated autophagy liberates amino acids in nutrient-deprived cancers to support tumour growth [49]. In addition, this PERK-ATF4 axis induces VEGF (vascular endothelial growth factor) and downregulates inhibitors of angiogenesis, so is important in maintaining tumour vascularity. Consequently, inhibition of PERK decreases tumour blood supply and reduces the growth of xenograft tumours in mice [45, 46]. Phosphorylation of eIF2 $\alpha$ is broadly cytoprotective, so its dephosphorylation might reasonably be predicted to contribute to tumour cell death. Accordingly, in murine medulloblastoma, loss of GADD34 enhances eIF2 $\alpha$ phosphorylation, thus promoting tumour growth, invasiveness and angiogenesis [50]. We showed in malignant mesothelioma that low expression of GADD34 correlates with increased tumour aggression [9]. It is therefore possible that agents that inhibit PERK or restore GADD34 function might prove beneficial in treating thoracic malignancy.

\section{Pulmonary fibrosis}

Up to $30 \%$ of cases of pulmonary fibrosis may have a genetic basis [51]. Classical genetics identified disease-associated mutations in several genes expressed by the type II alveolar epithelial cell (AECII). Some of these encode secretory proteins, for example mutations in SFTPA2 or SFTPC (surfactant proteins A2 or 
C, respectively) have been linked with interstitial lung disease of the usual interstitial pneumonitis and nonspecific interstitial pneumonitis patterns [52-57]. When expressed in cultured cells, some of these mutant proteins trigger the UPR. Prompted by this, ER stress was sought in lung tissue from patients with pulmonary fibrosis $[58,59]$. UPR activation was detected in the AECII of individuals with the L188Q SFTPC mutation and also in the alveolar epithelium of patients with sporadic idiopathic pulmonary fibrosis. Several factors linked with an increased likelihood of pulmonary fibrosis, e.g. viral infection, asbestos, amiodarone and old age, each activate the UPR in the lung, which is consistent with a potential role for ER stress in the pathogenesis of pulmonary fibrosis [10-14, 60]. However, the link between ER stress and interstitial lung disease appears to be complex. When L188Q SFTPC is expressed in murine type II pneumocytes the UPR is triggered, but this fails to cause pulmonary fibrosis [10]. However, mice expressing L188Q SFTPC show exaggerated pulmonary fibrosis when challenged with bleomycin. Likewise, co-administration of bleomycin and the ER stress-inducing drug tunicamycin enhances the degree of fibrosis [10].

Although many studies have focused on the alveolar epithelium, the precise cell types in which ER stress might play a role are unclear. Mutation of the COPA gene, which encodes a protein involved in Golgi-to-ER retrieval was found to cause autoimmune arthritis and pulmonary interstitial lung disease, but although UPR activation was detected in the lung epithelium the mutation caused a multisystem disorder of altered immune function [15]. In two separate studies, the UPR transcription factor CHOP has been identified as a potential driver of fibrosis $[16,61]$. Deletion of Chop in mice reduced alveolar epithelial apoptosis following bleomycin treatment, but in one of the studies adoptive transfer of wildtype macrophages restored normal levels of pulmonary fibrosis [16]. This suggests that modulation of the innate immune response by $\mathrm{CHOP}$ in the macrophage may play an important role, at least in the bleomycin model of fibrosis.

Finally, a role for epithelium-to-mesenchyme transition (EMT) in pulmonary fibrosis has been mooted repeatedly, but remains controversial. It is therefore worth noting that ER stress is a well-recognised inducer of EMT in the lung. Expression of mutant SFTPC, for example in primary murine AECII or AECII-like cell lines triggers morphological and biochemical EMT, and similar changes occur following treatment with chemical inducers of ER stress [17].

\section{Conclusion}

ER stress has been detected in several pulmonary diseases, although mechanistic understanding is often lacking. In cancer and pulmonary fibrosis there are, perhaps, sufficient data to support an important role for the UPR in modulating pathogenesis. Now that small molecules are available to target the UPR, it will be important to test their effects in models of these diseases.

\section{References}

1 Chambers JE, Marciniak SJ. Cellular mechanisms of endoplasmic reticulum stress signaling in health and disease. 2. Protein misfolding and ER stress. Am J Physiol Cell Physiol 2014; 307: C657-C670.

2 Marciniak SJ, Ron D. Endoplasmic reticulum stress signaling in disease. Physiol Rev 2006; 86: 1133-1149.

3 Mihailidou C, Panagiotou C, Kiaris H, et al. Crosstalk between C/EBP homologous protein (CHOP) and glucocorticoid receptor in lung cancer. Mol Cell Endocrinol 2016; 436: 211-223.

4 Zhang J, Liang Y, Lin Y, et al. IRE1 $\alpha$-TRAF2-ASK1 pathway is involved in CSTMP-induced apoptosis and ER stress in human non-small cell lung cancer A549 cells. Biomed Pharmacother 2016; 82: 281-289.

5 Fucikova J, Becht E, Iribarren K, et al. Calreticulin expression in human non-small cell lung cancers correlates with increased accumulation of antitumor immune cells and favorable prognosis. Cancer Res 2016; 76: 1746-1756.

6 Ramadori G, Konstantinidou G, Venkateswaran N, et al. Diet-induced unresolved ER stress hinders KRAS-driven lung tumorigenesis. Cell Metab 2015; 21: 117-125.

7 Yan J, Zhong N, Liu G, et al. Usp9x- and Noxa-mediated Mcl-1 downregulation contributes to pemetrexed-induced apoptosis in human non-small-cell lung cancer cells. Cell Death Dis 2014; 5: e1316.

8 Zismanov V, Drucker L, Gottfried M. ER homeostasis and motility of NSCLC cell lines can be therapeutically targeted with combined Hsp90 and HDAC inhibitors. Pulm Pharmacol Ther 2013; 26: 388-394.

9 Dalton LE, Clarke HJ, Knight J, et al. The endoplasmic reticulum stress marker CHOP predicts survival in malignant mesothelioma. Br J Cancer 2013; 108: 1340-1347.

10 Lawson WE, Cheng DS, Degryse AL, et al. Endoplasmic reticulum stress enhances fibrotic remodeling in the lungs. Proc Natl Acad Sci USA 2011; 108: 10562-7.

11 Ryan AJ, Larson-Casey JL, He C, et al. Asbestos-induced disruption of calcium homeostasis induces endoplasmic reticulum stress in macrophages. J Biol Chem 2014; 289: 33391-33403.

12 Mahavadi P, Henneke I, Ruppert C, et al. Altered surfactant homeostasis and alveolar epithelial cell stress in amiodarone-induced lung fibrosis. Toxicol Sci 2014; 142: 285-297.

13 Baek HA, Kim DS, Park HS, et al. Involvement of endoplasmic reticulum stress in myofibroblastic differentiation of lung fibroblasts. Am J Respir Cell Mol Biol 2012; 46: 731-739.

14 Bueno M, Lai YC, Romero Y, et al. PINK1 deficiency impairs mitochondrial homeostasis and promotes lung fibrosis. J Clin Invest 2015; 125: 521-538.

15 Watkin LB, Jessen B, Wiszniewski W, et al. COPA mutations impair ER-Golgi transport and cause hereditary autoimmune-mediated lung disease and arthritis. Nat Genet 2015; 47: 654-660. 
16 Yao Y, Wang Y, Zhang Z, et al. Chop deficiency protects mice against bleomycin-induced pulmonary fibrosis by attenuating M2 macrophage production. Mol Ther 2016; 24: 915-925.

17 Zhong Q, Zhou B, Ann DK, et al. Role of endoplasmic reticulum stress in epithelial-mesenchymal transition of alveolar epithelial cells: effects of misfolded surfactant protein. Am J Respir Cell Mol Biol 2011; 45: 498-509.

18 Ayaub EA, Kolb PS, Mohammed-Ali Z, et al. GRP78 and CHOP modulate macrophage apoptosis and the development of bleomycin-induced pulmonary fibrosis. J Pathol 2016; 239: 411-425.

19 Hoffman SM, Tully JE, Nolin JD, et al. Endoplasmic reticulum stress mediates house dust mite-induced airway epithelial apoptosis and fibrosis. Respir Res 2013; 14: 141.

20 Weichert N, Kaltenborn E, Hector A, et al. Some ABCA3 mutations elevate ER stress and initiate apoptosis of lung epithelial cells. Respir Res 2011; 12: 4.

21 van't Wout EF, van Schadewijk A, van Boxtel R, et al. Virulence factors of Pseudomonas aeruginosa induce both the unfolded protein and integrated stress responses in airway epithelial cells. PLoS Pathog 2015; 11: el004946.

22 Hrincius ER, Liedmann S, Finkelstein D, et al. Acute lung injury results from innate sensing of viruses by an ER stress pathway. Cell Rep 2015; 11: 1591-1603.

23 Hassan IH, Zhang MS, Powers LS, et al. Influenza A viral replication is blocked by inhibition of the inositol-requiring enzyme 1 (IRE1) stress pathway. J Biol Chem 2012; 287: 4679-4689.

24 Roberson EC, Tully JE, Guala AS, et al. Influenza induces endoplasmic reticulum stress, caspase-12-dependent apoptosis, and c-Jun $\mathrm{N}$-terminal kinase-mediated transforming growth factor- $\beta$ release in lung epithelial cells. $\mathrm{Am}$ J Respir Cell Mol Biol 2012; 46: 573-581.

25 Geraghty P, Baumlin N, Salathe MA, et al. Glutathione peroxidase-1 suppresses the unfolded protein response upon cigarette smoke exposure. Mediators Inflamm 2016; 2016: 9461289.

26 Kenche $\mathrm{H}$, Ye ZW, Vedagiri K, et al. Adverse outcomes associated with cigarette smoke radicals related to damage to protein-disulfide isomerase. J Biol Chem 2016; 291: 4763-4778.

27 Kenche H, Baty CJ, Vedagiri K, et al. Cigarette smoking affects oxidative protein folding in endoplasmic reticulum by modifying protein disulfide isomerase. FASEB J 2013; 27: 965-977.

28 Somborac-Bacura A, van der Toorn M, Franciosi L, et al. Cigarette smoke induces endoplasmic reticulum stress response and proteasomal dysfunction in human alveolar epithelial cells. Exp Physiol 2013; 98: 316-325.

29 Lee KS, Jeong JS, Kim SR, et al. Phosphoinositide 3-kinase- $\delta$ regulates fungus-induced allergic lung inflammation through endoplasmic reticulum stress. Thorax 2016; 71: 52-63.

30 Makhija L, Krishnan V, Rehman R, et al. Chemical chaperones mitigate experimental asthma by attenuating endoplasmic reticulum stress. Am J Respir Cell Mol Biol 2014; 50: 923-931.

31 Kim SR, Kim DI, Kang MR, et al. Endoplasmic reticulum stress influences bronchial asthma pathogenesis by modulating nuclear factor $\mathrm{\kappa B}$ activation. J Allergy Clin Immunol 2013; 132: 1397-1408.

32 Hsu KJ, Turvey SE. Functional analysis of the impact of ORMDL3 expression on inflammation and activation of the unfolded protein response in human airway epithelial cells. Allergy Asthma Clin Immunol 2013; 9: 4.

33 Tang AC, Saferali A, He G, et al. Endoplasmic reticulum stress and chemokine production in cystic fibrosis airway cells: regulation by STAT3 modulation. J Infect Dis 2017; 215: 293-302.

34 Blohmke CJ, Mayer ML, Tang AC, et al. Atypical activation of the unfolded protein response in cystic fibrosis airway cells contributes to p38 MAPK-mediated innate immune responses. J Immunol 2012; 189: 5467-5475.

35 Koyama M, Furuhashi M, Ishimura S, et al. Reduction of endoplasmic reticulum stress by 4-phenylbutyric acid prevents the development of hypoxia-induced pulmonary arterial hypertension. Am J Physiol Heart Circ Physiol 2014; 306: H1314-H1323.

36 Lu HY, Zhang J, Wang QX, et al. Activation of the endoplasmic reticulum stress pathway involving CHOP in the lungs of rats with hyperoxia-induced bronchopulmonary dysplasia. Mol Med Rep 2015; 12: 4494-4500.

37 Choo-Wing R, Syed MA, Harijith A, et al. Hyperoxia and interferon- $\gamma$-induced injury in developing lungs occur via cyclooxygenase-2 and the endoplasmic reticulum stress-dependent pathway. Am J Respir Cell Mol Biol 2013; 48: 749-757.

38 Marciniak SJ, Garcia-Bonilla L, Hu J, et al. Activation-dependent substrate recruitment by the eukaryotic translation initiation factor 2 kinase PERK. J Cell Biol 2006; 172: 201-209.

39 Lu PD, Jousse C, Marciniak SJ, et al. Cytoprotection by pre-emptive conditional phosphorylation of translation initiation factor 2. EMBO J 2004; 23: 169-179.

40 Marciniak SJ, Yun CY, Oyadomari S, et al. CHOP induces death by promoting protein synthesis and oxidation in the stressed endoplasmic reticulum. Genes Dev 2004; 18: 3066-3077.

41 Harding HP, Zhang $\mathrm{Y}$, Zeng $\mathrm{H}$, et al. An integrated stress response regulates amino acid metabolism and resistance to oxidative stress. Mol Cell 2003; 11: 619-633.

42 Chambers JE, Dalton LE, Clarke HJ, et al. Actin dynamics tune the integrated stress response by regulating eukaryotic initiation factor $2 \alpha$ dephosphorylation. eLife 2015; 4: e04872.

43 Pennuto M, Tinelli E, Malaguti M, et al. Ablation of the UPR-mediator CHOP restores motor function and reduces demyelination in Charcot-Marie-Tooth 1B mice. Neuron 2008; 57: 393-405.

44 Steiling K, van den Berge M, Hijazi K, et al. A dynamic bronchial airway gene expression signature of chronic obstructive pulmonary disease and lung function impairment. Am J Respir Crit Care Med 2013; 187: 933-942.

45 Clarke HJ, Chambers JE, Liniker E, et al. Endoplasmic reticulum stress in malignancy. Cancer Cell 2014; 25: 563-573.

46 Atkins C, Liu Q, Minthorn E, et al. Characterization of a novel PERK kinase inhibitor with antitumor and antiangiogenic activity. Cancer Res 2013; 73: 1993-2002.

47 Bi M, Naczki C, Koritzinsky M, et al. ER stress-regulated translation increases tolerance to extreme hypoxia and promotes tumor growth. EMBO J 2005; 24: 3470-3481.

48 Rouschop KM, Dubois LJ, Keulers TG, et al. PERK/eIF2 $\alpha$ signaling protects therapy resistant hypoxic cells through induction of glutathione synthesis and protection against ROS. Proc Natl Acad Sci USA 2013; 110: 4622-4627.

49 Rouschop KM, van den Beucken T, Dubois L, et al. The unfolded protein response protects human tumor cells during hypoxia through regulation of the autophagy genes MAP1LC3B and ATG5. J Clin Invest 2010; 120: 127-141.

50 Lin $\mathrm{W}$, Lin $\mathrm{Y}, \mathrm{Li}$ J, et al. A deregulated integrated stress response promotes interferon- $\gamma$-induced medulloblastoma. J Neurosci Res 2011; 89: 1586-1595. 
51 Mathai SK, Yang IV, Schwarz MI, et al. Incorporating genetics into the identification and treatment of idiopathic pulmonary fibrosis. BMC Med 2015; 13: 191.

52 Nogee LM, Dunbar AE 3rd, Wert SE, et al. A mutation in the surfactant protein C gene associated with familial interstitial lung disease. N Engl J Med 2001; 344: 573-579.

53 Thomas AQ, Lane K, Phillips J 3rd, et al. Heterozygosity for a surfactant protein C gene mutation associated with usual interstitial pneumonitis and cellular nonspecific interstitial pneumonitis in one kindred. Am J Respir Crit Care Med 2002; 165: 1322-1328.

54 Chibbar R, Shih F, Baga M, et al. Nonspecific interstitial pneumonia and usual interstitial pneumonia with mutation in surfactant protein C in familial pulmonary fibrosis. Mod Pathol 2004; 17: 973-980.

55 Brasch F, Griese M, Tredano M, et al. Interstitial lung disease in a baby with a de novo mutation in the SFTPC gene. Eur Respir J 2004; 24: 30-39.

56 Wang Y, Kuan PJ, Xing C, et al. Genetic defects in surfactant protein A2 are associated with pulmonary fibrosis and lung cancer. Am J Hum Genet 2009; 84: 52-59.

57 Maitra M, Wang Y, Gerard RD, et al. Surfactant protein A2 mutations associated with pulmonary fibrosis lead to protein instability and endoplasmic reticulum stress. J Biol Chem 2010; 285: 22103-22113.

58 Korfei M, Ruppert C, Mahavadi P, et al. Epithelial endoplasmic reticulum stress and apoptosis in sporadic idiopathic pulmonary fibrosis. Am J Respir Crit Care Med 2008; 178: 838-846.

59 Lawson WE, Crossno PF, Polosukhin VV, et al. Endoplasmic reticulum stress in alveolar epithelial cells is prominent in IPF: association with altered surfactant protein processing and herpesvirus infection. Am J Physiol Lung Cell Mol Physiol 2008; 294: L1119-L1126.

60 Mulugeta S, Nguyen V, Russo SJ, et al. A surfactant protein C precursor protein BRICHOS domain mutation causes endoplasmic reticulum stress, proteasome dysfunction, and caspase 3 activation. Am J Respir Cell Mol Biol 2005; 32: 521-530.

61 Tanaka Y, Ishitsuka Y, Hayasaka M, et al. The exacerbating roles of CCAAT/enhancer-binding protein homologous protein (CHOP) in the development of bleomycin-induced pulmonary fibrosis and the preventive effects of tauroursodeoxycholic acid (TUDCA) against pulmonary fibrosis in mice. Pharmacol Res 2015; 99: 52-62. 